\title{
Prognosis of adult patients with bacteremia caused by extensively resistant Acinetobacter baumannii
}

\author{
Yu-Chen Tseng ${ }^{\mathrm{a}, 1}$, Jann-Tay Wang ${ }^{\mathrm{c}, 1}, \mathrm{Fe}-\mathrm{Lin}$ Lin $\mathrm{Wu}^{\mathrm{a}}$, \\ Yee-Chun Chen ${ }^{\mathrm{c}}$, Wei-Chu Chie ${ }^{\mathrm{b}}$, Shan-Chwen Chang ${ }^{\mathrm{a}, \mathrm{c}, *}$ \\ ${ }^{a}$ Graduate Institute of Clinical Pharmacy, College of Medicine, National Taiwan University, Taipei 100, Taiwan \\ ${ }^{\mathrm{b}}$ Graduate Institute of Preventive Medicine, College of Public Health, National Taiwan University, Taipei 100, Taiwan \\ ${ }^{\mathrm{c}}$ Division of Infectious Diseases, Department of Internal Medicine, National Taiwan University Hospital, Taipei 100, Taiwan \\ Received 17 January 2007; accepted 29 April 2007
}

\begin{abstract}
Nosocomial bacteremia caused by extensively resistant Acinetobacter baumannii (ERAB) is an emerging problem in Taiwanese hospitals. Accordingly, we retrospectively investigated the epidemiology and outcomes of adult patients with ERAB bacteremia (ERABB) after different treatments from January 2001 to September 2004 at the National Taiwan University Hospital, Taipei, Taiwan. A total of 56 adult patients with ERABB and without other simultaneous infections were enrolled. Demographic, clinical, and laboratory data were obtained from medical records. Clinical data included underlying diseases and conditions with onset within 7 days after ERABB, severity of ERABB, antibiotic regimens for ERABB, and clinical outcomes. Laboratory data included hemograms, liver function tests, renal function tests, and albumin levels. Coagulation profiles were obtained at ERABB onset and 7 days before and after onset. All 56 episodes of ERABB were hospital-acquired. Most patients had Acute Physiology Scores and Chronic Health Evaluation II scores $\geq 17$ (66.1\%) and Pitt bacteremia score $\geq 4(66.1 \%)$. Many had comorbid diseases at ERABB onset. Crude mortality rates on days 2, 7, 15, 30, and at discharge were 23.2\%, $30.4 \%, 37.5 \%, 48.2 \%$, and $60.7 \%$, respectively. High severity of ERABB (Pitt bacteremia score $\geq 4$ ) and presence of immunosuppression were the only 2 predictors for day 30 mortality (odds ratios of 18.53 and 8.06, respectively). Antibiotic regimens for ERABB did not have a significant influence on ERABB outcomes.
\end{abstract}

(C) 2007 Elsevier Inc. All rights reserved.

Keywords: Acinetobacter baumannii; Antibiotic resistance; Bacteremia; Prognosis; Antibiotics

\section{Introduction}

Acinetobacter spp. are aerobic, encapsulated, nonmotile, nonfermentative, Gram-negative coccobacilli with low virulence (Lai et al., 1999; Chang et al., 1995; Kuo et al., 2003; Wang et al., 2003; Allen et al., 2000). Free-living Acinetobacter can be found on both animate and inanimate objects (Allen et al., 2000), colonize humans, survive on dry inanimate objects for several days, and emerge as important

* Corresponding author. Division of Infectious Diseases, Department of Internal Medicine, National Taiwan University Hospital, Taipei 100, Taiwan. Tel.: +886-2-23123456x5401; fax: +886-2-23958721.

E-mail address: changsc@ntu.edu.tw (S.-C. Chang).

${ }^{1}$ Yu-Chen Tseng and Jann-Tay Wang equally contributed in this article. opportunistic pathogens, especially among critically ill patients (Manikal et al., 2000).

Nosocomial infections caused by Acinetobacter baumannii have increased in recent years, especially in patients staying in intensive care units (ICU) (Cisneros et al., 1996) and in immunosuppressed hosts (Levin et al., 2003; Seifert et al., 1995) or burn patients (Cisneros et al., 1996). Although there are significant differences in antimicrobial resistance patterns for $A$. baumannii demonstrated in various studies, the overall trend is toward increasing resistance (Allen et al., 2000). Therefore, the antibiotics of choice for treating A.baumannii infection have become more and more limited (Lai et al., 1999; Chang et al., 1995; Kuo et al., 2003).

A strain of extensively resistant $A$. baumannii (ERAB), which is resistant to all currently available antipseudomonal antibiotics available in Taiwan, has emerged rapidly at the 
National Taiwan University Hospital (NTUH), Taipei, Taiwan, since 1998 (Hsueh et al., 2002a, 2002b). Since then, ERAB was found in many hospitals in Taiwan (Center of Diseases Control, Taiwan; unpublished data) and caused some nosocomial outbreaks (Wang et al., 2003). Among various infections caused by ERAB, bacteremia is of special concern because of its high mortality (Kuo et al., 2003). However, prognostic factors and outcomes of patients with ERAB bacteremia (ERABB) have not been well studied (Kuo et al., 2003; Lee et al., 2005). Furthermore, the most effective antibiotic regimen for treatment of ERABB has not been determined.

Therefore, our study was designed to identify prognostic factors and compare treatment effects of different antibiotic regimens used for treating ERABB.

\section{Patients and methods}

\subsection{Hospital settings and patients}

NTUH, which is in northern Taiwan, is a major teaching hospital with a total capacity of 2200 beds. It offers both primary and tertiary care for patients with acute diseases. Since early 1980s at NTUH, there has been a well-trained and organized infection control team in charge of hospitalwide surveillance for nosocomial infections and resistant pathogens isolated from all clinical specimens.

From January 2001 to September 2004, all hospitalized adult patients (aged $\geq 18$ years) at NTUH who had ERABB were enrolled in our study. Patients who had other microorganisms isolated concomitantly from blood or other infections caused by any microorganisms other than ERAB within 2 days of onset of ERABB were excluded.

\subsection{Definition of ERAB and ERABB}

ERAB was defined as an isolate of $A$. baumannii resistant to trimethoprim/sulfamethoxazole, piperacillin/tazobactam, aztreonam, ceftazidime, ceftriaxone, cefepime, ciprofloxacin, levofloxacin, gentamicin, amikacin, and meropenem determined by disc diffusion method (National Committee For Clinical Laboratory Standards, 2000).

At NTUH, the blood culture system used is Bactec 2-bottle culture system (Becton Dickinson, Maryland). Patients with ERABB was defined as who had clinical symptoms and signs of infection (such as fever, leukocytosis, elevated C-reactive protein [CRP], or sepsis) and one or more positive blood culture of ERAB at the same time. Primary ERABB was defined as ERABB without an identifiable infection focus or those due to catheter-related infection (Wang et al., 2004a, 2004b).

Nosocomial bacteremia was defined as bacteremia that occurred more than $48 \mathrm{~h}$ after hospital admission or bacteremia that occurred less than $48 \mathrm{~h}$ after admission to the hospital in patients who had been hospitalized in any hospital within 2 weeks before admission (Lautenbach et al., 2001).

\subsection{Definition of organ failure and dysfunction}

Heart failure was defined according to criteria proposed by Ho et al. (1993). Respiratory failure was defined as partial pressure of oxygen of arterial blood $<60 \mathrm{~mm} \mathrm{Hg}$ with a fraction of inspired oxygen $>0.5$, or alveolar-arterial oxygen tension difference $>300 \mathrm{~mm} \mathrm{Hg}$ with a fraction of inspired oxygen of $>0.5$, or requirement for mechanical ventilation (Garcia-Garmendia et al., 2001). Abnormal liver function was defined as $>5$ consecutive positive tests for elevated aspartate aminotransferase (AST) and/or alanine aminotransferase (ALT) and the peak level being greater than 5 times of the normal limit (for AST, $36 \mathrm{U} / \mathrm{L}$ for male patient and $30 \mathrm{U} / \mathrm{L}$ for female patient; for ALT, $40 \mathrm{U} / \mathrm{L}$ for male patient and $30 \mathrm{U} / \mathrm{L}$ for female patient). Hepatic failure was defined as serum bilirubin $>3.0 \mathrm{mg} / \mathrm{dL}$ and prothrombin time $>15 \mathrm{~s}$, or biopsy evidence of cirrhosis or necrosis (Linden et al., 1997). Cirrhosis was considered as a definite diagnosis if histologic evidence was present or a probable diagnosis if clinical and analytical data suggested chronic liver disease, hepatocellular dysfunction, and portal hypertension (Caballero-Granado et al., 2001). Acute renal failure was defined as an increase of $0.5 \mathrm{mg} / \mathrm{dL}$ in normal serum creatinine levels or of $1 \mathrm{mg} / \mathrm{dL}$ in abnormal serum creatinine levels. Chronic renal insufficiency was defined as a creatinine level in plasma $>2 \mathrm{mg} / \mathrm{dL}$ and a medical record that suggested that it was a chronic disturbance (i.e., confirmation of its previous existence, normocytic normochromic anemia, renal atrophy, and renal osteodystrophy) (Caballero-Granado et al., 2001). Chronic renal failure was defined as chronic renal disease progression to the point where creatinine clearance was $<10 \mathrm{~mL} / \mathrm{min}$.

\subsection{Study design and data collection}

This is a retrospective cohort study. A standardized case record form was used to collect patients' demographic, clinical, and laboratory data. The clinical data consisted of 1) underlying diseases, 2) invasive procedures before the onset of ERABB, 3) severity of the underlying condition at ERABB onset using the Acute Physiology and Chronic Health Evaluation II (APACHE II) score (Knaus et al., 1985), 4) severity of ERABB by Pitt bacteremia score $(\geq 4)$ and the presence of septic shock according to the classification recommended by the American College of Chest Physicians/ Society of Critical Care Medicine (The American College of Chest Physicians/Society of Critical Medicine, 1992; Chow and $\mathrm{Yu}, 1999)$, 5) treatment regimen for ERABB, 6) complications related to ERABB, and 7) outcome.

Immunosuppression was defined as the presence of prior organ transplantation, human immunodeficiency virus infection, and immunosuppressive therapy, which included antineoplastic therapy within 6 weeks of onset of ERABB (Tilley and Roberts, 1994), corticosteroids at a dose equivalent to or higher than $20 \mathrm{mg}$ of prednisolone daily for at least 2 weeks or $30 \mathrm{mg}$ of prednisolone daily for at least 1 week before a positive blood culture for ERAB (Esel et al., 
Table 1

Demographic data and clinical conditions for patients $(n=56)$ with ERABB

\begin{tabular}{|c|c|c|c|c|c|c|}
\hline Variable & No. & $(\%)$ & Mean & $\mathrm{SD}$ & Median & Range \\
\hline Age (years) & & & 65.5 & 16.0 & 68 & $20-92$ \\
\hline Sex: male/female & $30 / 26$ & $(53.6 / 46.4)$ & & & & \\
\hline \multicolumn{7}{|l|}{ Ward at ERABB onset } \\
\hline General ward & 16 & $(28.6)$ & & & & \\
\hline Emergency room & 1 & $(1.8)$ & & & & \\
\hline ICU & 39 & $(69.6)$ & & & & \\
\hline ICU experience before ERABB & 48 & $(85.7)$ & & & & \\
\hline \multicolumn{7}{|l|}{ Underlying disease } \\
\hline Cerebrovascular accident & 13 & $(23.2)$ & & & & \\
\hline Diabetes mellitus & 20 & $(35.7)$ & & & & \\
\hline Cardiovascular diseases & 32 & $(57.1)$ & & & & \\
\hline Hypertension & 26 & $(46.4)$ & & & & \\
\hline Congestive heart failure & 10 & $(17.9)$ & & & & \\
\hline Coronary artery disease & 15 & $(26.8)$ & & & & \\
\hline VHD & 4 & $(7.1)$ & & & & \\
\hline Others & 1 & $(1.8)$ & & & & \\
\hline Shock & 21 & $(37.5)$ & & & & \\
\hline Respiratory diseases & 46 & $(82.1)$ & & & & \\
\hline COPD & 7 & $(12.5)$ & & & & \\
\hline Respiratory failure & 45 & $(80.4)$ & & & & \\
\hline Hepatobiliary diseases & 19 & $(33.3)$ & & & & \\
\hline Hepatic failure/cirrhosis & 5 & $(8.9)$ & & & & \\
\hline Renal diseases & 23 & $(41.1)$ & & & & \\
\hline End-stage renal disease & 7 & $(12.5)$ & & & & \\
\hline Dialysis dependence & 16 & $(28.6)$ & & & & \\
\hline Autoimmune diseases & 5 & $(8.9)$ & & & & \\
\hline Malignancy & 19 & $(33.9)$ & & & & \\
\hline Solid tumor & 11 & (19.6) & & & & \\
\hline Hematologic malignancy & 8 & $(14.3)$ & & & & \\
\hline Immunologically suppressed? & 24 & $(42.9)$ & & & & \\
\hline \multicolumn{7}{|l|}{ SIRS criteria at ERABB onset } \\
\hline Without septic shock & 34 & $(60.7)$ & & & & \\
\hline With septic shock & 22 & $(39.3)$ & & & & \\
\hline Pitt bacteremia score at ERABB onset & & & 4.34 & 2.56 & 4 & $0-14$ \\
\hline Critical (Pitt score $\geq 4$ ) & 37 & $(66.1)$ & & & & \\
\hline APACHE II score at ERABB onset & & & 20.8 & 9.3 & 19 & $6-39$ \\
\hline$\geq 17$ & 37 & $(66.1)$ & & & & \\
\hline DIC at ERABB onset & 11 & $(19.6)$ & & & & \\
\hline WBC count & 55 & $(98.2)$ & 11864 & 8475 & 9400 & $20-39310$ \\
\hline Neutropenia & 3 & $(5.4)$ & & & & \\
\hline Leukocytosis & 26 & $(47.3)$ & & & & \\
\hline Hemoglobin (g/dL) (data for 55 patients) & & & 9.86 & 1.71 & 9.7 & $6.5-17.2$ \\
\hline Anemia & 9 & $(16.4)$ & & & & \\
\hline Albumin (g/dL) (35 patients) & 35 & $(62.5)$ & 2.57 & 0.45 & 2.80 & $1.62-3.98$ \\
\hline CRP (mg/dL) (28 patients) & & & 8.84 & 4.90 & 8.85 & $1.03-21.2$ \\
\hline $\mathrm{CRP} \geq 5$ & 21 & $(75.0)$ & & & & \\
\hline Scr (mg/dL) (51 patients) & & & 1.88 & 1.53 & 1.20 & $0.23-6.67$ \\
\hline Elevated $(>1.5 \mathrm{mg} / \mathrm{dL})$ & 23 & $(45.1)$ & & & & \\
\hline LDH (8 patients data) & & & 761.5 & 813.81 & 515.5 & $238-2753$ \\
\hline Elevated (>460 U/L) & 6 & $(75)$ & & & & \\
\hline Total bilirubin (37 patients) & & & 8.05 & 18.72 & 1.22 & $0.25-109.00$ \\
\hline Elevated $(>1.5 \mathrm{mg} / \mathrm{dL})$ & 17 & $(45.9)$ & & & & \\
\hline ALT (only 22 patients had data) & & & 89.81 & 259.73 & 29.5 & $10-1248$ \\
\hline Elevated $(>40 \mathrm{U} / \mathrm{L})$ & 8 & $(36.4)$ & & & & \\
\hline \multicolumn{7}{|l|}{ Infection source of ERABB } \\
\hline Primary ERABB & 39 & $(69.6)$ & & & & \\
\hline No identifiable source & 32 & $(57.1)$ & & & & \\
\hline $\mathrm{CVC}$ & 7 & $(12.5)$ & & & & \\
\hline Secondary — respiratory tract & 15 & $(26.8)$ & & & & \\
\hline Secondary-others & 2 & $(3.6)$ & & & & \\
\hline Total length of hospital stay (days) & & & 92.8 & 97.6 & 68 & $3-590$ \\
\hline
\end{tabular}


Table 1 (continued)

\begin{tabular}{|c|c|c|c|c|c|c|}
\hline Variable & No. & $(\%)$ & Mean & SD & Median & Range \\
\hline Before ERABB onset & & & 50.1 & 55.3 & 34 & $0-292$ \\
\hline After ERABB onset & & & 42.6 & 64.7 & 22.5 & $1-331$ \\
\hline \multicolumn{7}{|l|}{ Overall mortality } \\
\hline At 15 days after ERABB onset & 21 & $(37.5)$ & & & & \\
\hline At 30 days after ERABB onset & 27 & $(48.2)$ & & & & \\
\hline At discharge & 34 & $(60.7)$ & & & & \\
\hline
\end{tabular}

$\mathrm{COPD}=$ chronic obstructive pulmonary disease; $\mathrm{WBC}=$ white blood cell; $\mathrm{Scr}=$ serum creatinine concentration; $\mathrm{LDH}=$ lactate dehydrogenase; $\mathrm{ALT}=$ alanine transaminase; $\mathrm{VHD}=$ valvular heart disease

2003; Galofre et al., 1994), and other immunosuppressants such as azathioprine, cyclosporine, mycophenolate mofetil, tacrolimus, and sirolimus.

Invasive procedures included i) usage of central venous catheters (CVCs), arterial catheters, port A catheters, endotracheal tubes, nasogastric/nasoduodenal tubes, and peripheral venous catheters, thoracentesis, and paracentesis within 7 days before onset of ERABB; ii) simple operation under local anesthesia within 7 days before onset of ERABB; and iii) operations under general anesthesia within 30 days previous to onset of ERABB (Levin et al., 2003).

The severity of ERABB was defined as critical if the Pitt bacteremia score was equal to or greater than 4 . The severity of patients' clinical condition was defined as severe if the APACHE II score was equal to or greater than 17 (Chow and Yu, 1999; Smolyakov et al., 2003). New organ failure was defined as that which developed within 7 days of ERABB onset, and new bacterial infection was defined as that which occurred within 30 days after onset of ERABB.

Antibiotics regimens used for ERABB were evaluated at 2 different stages, that is, as initial or definite treatments. Initial treatment was the regimen used within $48 \mathrm{~h}$ after onset of ERABB, when the blood culture result was usually not available. Definite treatment was the regimen used after the availability of blood culture results. To evaluate the potential benefit of antibiotic treatment, both initial and definite treatments were further classified into 2 groups by 3 different classification methods. First, regimens containing ampicillin/ sulbactam were classified into one group and all others into another group. Second, regimens containing carbapenem (imipenem or meropenem) and ampicillin/sulbactam were classified into one group and all others into another group. Third, regimens containing both carbapenems (imipenem or meropenem) and aminoglycosides were classified into one group and all others into another group.

Laboratory data including hemogram, CRP, liver function test, renal function test, albumin level, and profile of disseminated intravascular coagulopathy (DIC) were evaluated before and after the onset of ERABB.

\subsection{Mortality of ERABB}

We evaluated all-cause mortality by days $2,7,15$, and 30 after the onset of ERABB, and at discharge. For patients who were discharged before day 30, clinical status was determined by the records of follow-up visits in outpatient clinics.

\subsection{Statistical analyses}

Statistical analyses were executed using SAS 9.1.3 (SAS Institute, Cary, NC). Continuous variables were described as mean \pm SD and median. They were compared using Student's $t$ test. Categoric variables were compared with a $\chi^{2}$ test or, if the expected values were below 10, a Fisher's exact test. Predictors for day 30 mortality were identified using logistic regression. All clinical and laboratory parameters were tested for univariate analysis first. The parameters with a $P$ value less than 0.2 and those with potentially biologic meanings would be put into the model of multivariate analysis. However, the parameters with collinearity [variance inflation factors (VIF) $>10$ determined by linear regression model using the option of VIF in SAS program] would not be put in the final model simultaneously. A stepwise model comparison and selection were used to determine the final model of multiple variables analysis. All tests were 2 tailed. A $P$ value less than 0.05 was considered statistically significant.

\section{Results}

During the study period, there were 59 cases that met the definition of ERABB. Three patients with missing medical records were excluded from the analysis.

The demographic, clinical, and laboratory data for the 56 patients with ERABB are listed in Table 1. All 56 episodes of ERABB were nosocomial. The male-to-female ratio was 1.2:1. The mean age was 65.5 years (range, 2092 years). Among them, 54 (96.4\%) had various underlying diseases. Thirty-nine (69.6\%) developed ERABB while in ICUs, 16 (28.6\%) while in the general wards, and 1 while in the emergency room. Of the 16 who developed ERABB in general wards, $8(50.0 \%)$ had stayed in an ICU before the onset of ERABB during the same hospitalization. The mean APACHE II scores for all 56 patients were $20.8 \pm 9.3$ (median, 19; range, 6-39). At the onset of ERABB, all 56 patients met the criteria for sepsis, and $22(39.3 \%)$ had septic shock. The Pitt bacteremia scores were $4.34 \pm 2.56$ (median, 4; range, 0-14). Twenty-four patients (42.9\%) were 

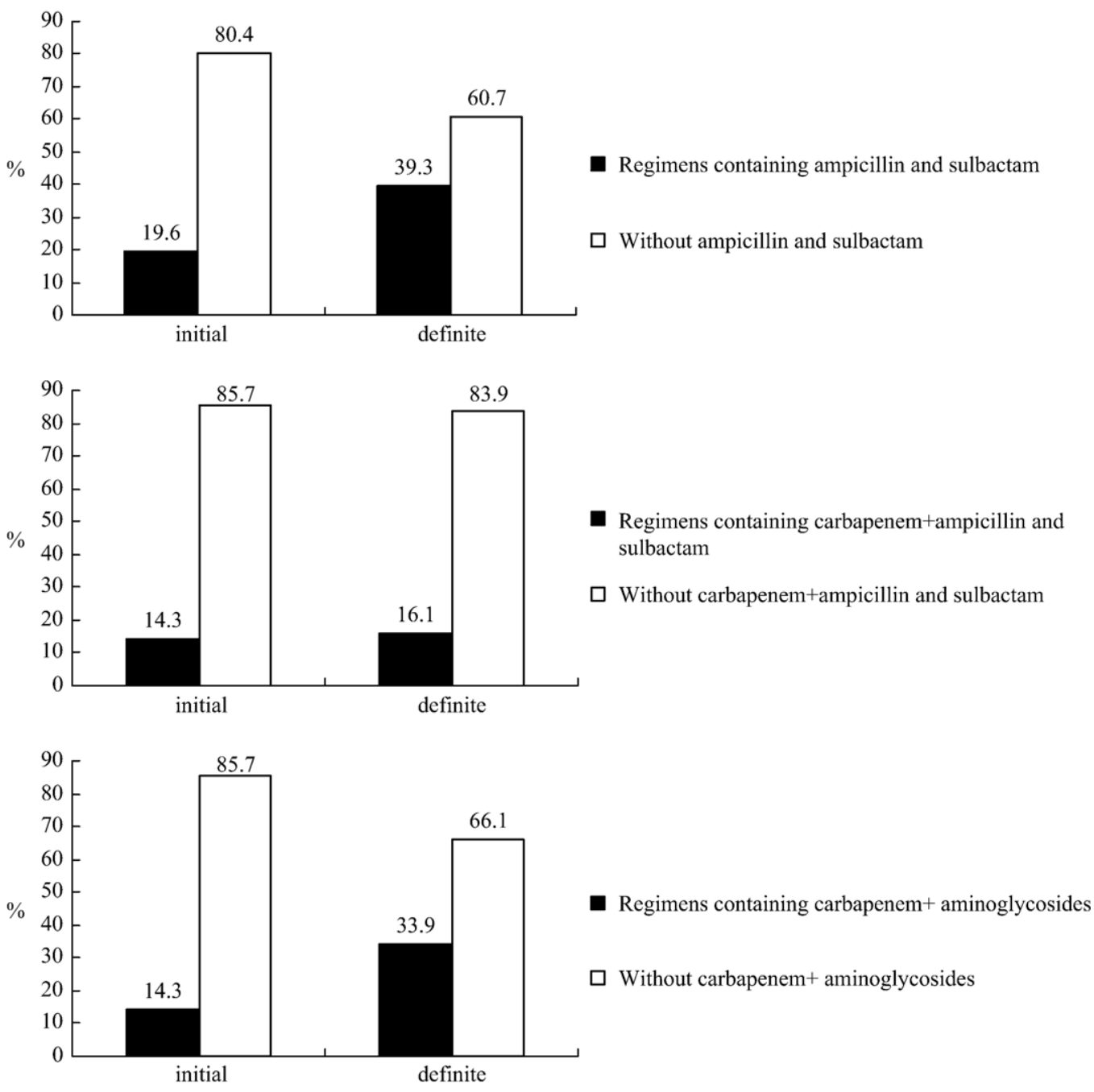

Fig. 1. Antibiotic regimens for ERABB patients as initial treatment and definite treatment.

classified as immunosuppressed hosts during ERABB onset. Among these 24 patients, 4 were recipients of organ transplantation, 2 received chemotherapy, and 19 received corticosteroids. The average length of total hospital stays was $92.8 \pm 97.6$ days (median, 68 days; range, 3-590 days). The average length of hospital stays before the onset of ERABB was $50.1 \pm 55.3$ days (median, 34 days; range, 0-292 days). The average length of hospital stays after the onset of ERABB was $42.6 \pm 67.4$ days (median, 22.5 days; range, $1-331$ days). Thirty-four patients $(60.7 \%)$ had died by the time of discharge.

Most ERABB episodes were classified as primary bacteremia (69.6\%). Eleven $(19.6 \%)$ of the 56 patients had DIC. Among the 55 patients whose hemograms were checked during the onset of ERABB, 3 (5.4\%) had neutropenia $(<1500 / \mu \mathrm{L}), 26(47.3 \%)$ had leukocytosis $(>11000 / \mu \mathrm{L})$, and 9 had anemia (hemoglobin $<11 \mathrm{~g} / \mathrm{dL}$ ). Fifty-one patients had available renal function test results, and $23(45.1 \%)$ of them had abnormal serum creatinine levels $(>1.5 \mathrm{mg} / \mathrm{dL})$. The serum level of alanine transami- nase was available for 22 patients, and 8 (36.4\%) of them were abnormal $(>40 \mathrm{U} / \mathrm{L})$. Thirty-seven patients had available serum bilirubin levels, and 17 (45.9\%) of them were abnormal. Other laboratory data are shown in Table 1.

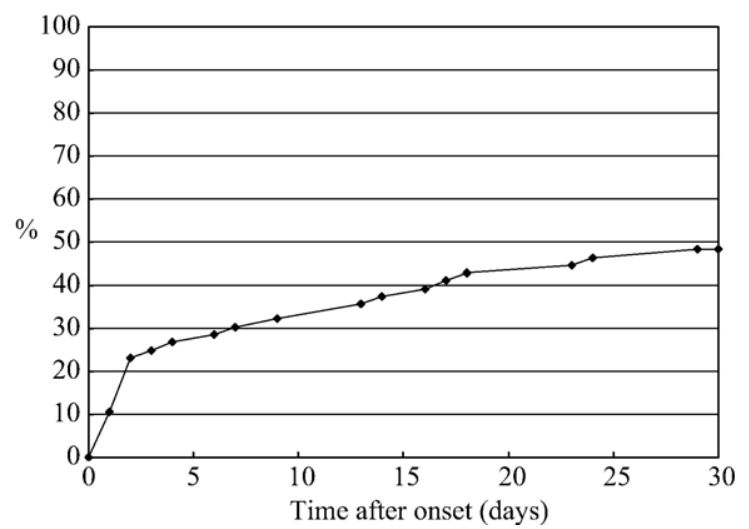

Fig. 2. Cumulative mortality rate of 56 patients with ERABB. 
Table 2

Comparative parameters and univariate analysis of mortality on day 30 for ERABB patients

\begin{tabular}{|c|c|c|c|c|c|c|c|c|}
\hline \multirow[t]{2}{*}{ Variables } & \multicolumn{2}{|c|}{ Alive, $n=29$} & \multicolumn{2}{|c|}{ Dead, $n=27$} & \multirow[t]{2}{*}{$P^{\mathrm{a}}$} & \multicolumn{3}{|c|}{ Logistic regression } \\
\hline & No. & $(\%)$ & No. & $(\%)$ & & OR & $95 \%$ confidence interval & $P$ \\
\hline Cerebrovascular accident & 9 & $(31.0)$ & 2 & (7.4) & 0.0420 & 0.18 & $0.03-0.92$ & 0.0391 \\
\hline Diabetes mellitus & 11 & (37.9) & 9 & $(33.3)$ & 0.7849 & 0.82 & $0.27-2.45$ & 0.7199 \\
\hline Cardiovascular diseases & 16 & $(55.2)$ & 16 & $(59.3)$ & 0.7928 & 1.18 & $0.41-3.41$ & 0.7578 \\
\hline Respiratory diseases & 20 & $(69.0)$ & 24 & $(88.9)$ & 0.1043 & 3.60 & $0.86-15.12$ & 0.0802 \\
\hline Respiratory failure & 17 & $(58.6)$ & 24 & $(88.9)$ & 0.0154 & 5.65 & $1.38-23.12$ & 0.0161 \\
\hline Abnormal liver function & 7 & $(24.1)$ & 13 & $(48.15)$ & 0.0940 & 2.918 & $0.936-9.099$ & 0.0649 \\
\hline Hepatic failure/cirrhosis & 1 & $(3.5)$ & 4 & $(14.8)$ & 0.1855 & 4.869 & $0.51-46.65$ & 0.1697 \\
\hline Abnormal renal function & 10 & $(34.5)$ & 17 & $(63.0)$ & 0.0601 & 3.23 & $1.08-9.64$ & 0.0357 \\
\hline Acute renal failure & 5 & $(17.2)$ & 14 & $(51.9)$ & 0.0104 & 5.17 & $1.52-17.58$ & 0.0085 \\
\hline Chronic renal insufficiency & 4 & $(13.8)$ & 3 & $(11.1)$ & 1.0000 & 0.76 & $0.16-3.86$ & 0.7621 \\
\hline Autoimmune diseases & 1 & $(3.5)$ & 4 & $(14.8)$ & 0.1855 & 4.87 & $0.51-46.65$ & 0.1697 \\
\hline Malignancy & 8 & $(27.6)$ & 11 & $(40.7)$ & 0.3991 & 1.81 & $0.59-5.53$ & 0.3011 \\
\hline Immunosuppression & 6 & $(20.7)$ & 18 & $(66.7)$ & $9.915 \mathrm{E} 04$ & 7.67 & $2.30-25.53$ & 0.0009 \\
\hline Transplantation recipient & 1 & $(3.5)$ & 3 & $(11.1)$ & 0.4558 & 2.91 & $0.40-21.38$ & 0.2942 \\
\hline No & 28 & $(36.6)$ & 24 & $(88.9)$ & & baseline & & \\
\hline Bone marrow & 1 & $(3.5)$ & 2 & $(7.4)$ & & 2.24 & $0.19-26.22$ & 0.5206 \\
\hline Heart & 0 & $(0.0)$ & 1 & $(3.7)$ & & - & - & - \\
\hline Immunosuppressive therapy & 5 & $(17.2)$ & 16 & $(59.3)$ & 0.0021 & 6.98 & $2.04-23.93$ & 0.0020 \\
\hline Chemotherapy & 1 & $(3.5)$ & 1 & $(3.7)$ & 1.0000 & 1.08 & $0.06-18.12$ & 0.9589 \\
\hline Steroid & 4 & $(13.8)$ & 15 & $(55.6)$ & 0.0016 & 7.81 & $2.13-28.67$ & 0.0019 \\
\hline DIC & 2 & $(6.9)$ & 9 & (33.3) & 0.0184 & 6.75 & $1.30-34.94$ & 0.0228 \\
\hline Neutropenia & 0 & $(0.0)$ & 3 & $(11.1)$ & 0.1055 & - & - & - \\
\hline Subsequent new infections & 14 & $(48.3)$ & 11 & $(40.7)$ & 0.6016 & 0.74 & $0.26-2.12$ & 0.5713 \\
\hline Not caused by ERAB & 9 & $(31.0)$ & 4 & $(14.8)$ & 0.2095 & 0.39 & $0.10-1.45$ & 0.1586 \\
\hline Caused by ERAB & 9 & $(31.0)$ & 8 & $(29.6)$ & 1.0000 & 0.94 & $0.30-2.93$ & 0.9091 \\
\hline Hemoglobin & & & & & 1.000 & 1.250 & $0.297-5.256$ & 0.7607 \\
\hline Hemoglobin $\leq 11$ & 23 & $(82.1)$ & 23 & $(85.2)$ & & & & \\
\hline Hemoglobin $>11$ & 5 & $(17.9)$ & 4 & $(14.8)$ & & & & \\
\hline Albumin & & & & & 0.0082 & 10.286 & $1.737-60.901$ & 0.0102 \\
\hline Albumin $\leq 2.5$ & 2 & $(10.0)$ & 8 & $(53.3)$ & & & & \\
\hline Albumin $>2.5$ & 18 & $(90.0)$ & 7 & $(46.7)$ & & & & \\
\hline \multicolumn{9}{|l|}{ Invasive procedure } \\
\hline Arterial catheters & 16 & $(55.2)$ & 23 & $(85.2)$ & 0.0205 & 4.672 & $1.287-16.964$ & 0.0191 \\
\hline CVCs & 24 & $(82.8)$ & 26 & $(96.3)$ & 0.1946 & 5.416 & $0.590-49.788$ & 0.1354 \\
\hline Swan-Ganz catheters & 2 & $(6.9)$ & 1 & $(3.7)$ & 1.0000 & 0.519 & $0.044-6.079$ & 0.6016 \\
\hline Urinary catheters/Foley & 19 & $(65.5)$ & 21 & $(77.8)$ & 0.3820 & 1.842 & $0.562-6.038$ & 0.3132 \\
\hline Endotracheal tubes/tracheal tubes & 18 & $(62.1)$ & 23 & $(85.2)$ & 0.0718 & 3.514 & $0.958-12.894$ & 0.0581 \\
\hline Nasogastric tube/duodenal tubes & 23 & $(79.3)$ & 23 & $(85.2)$ & 0.7308 & 1.500 & $0.373-6.028$ & 0.5678 \\
\hline Shunting surgery & 5 & $(17.2)$ & 10 & $(37.0)$ & 0.1334 & 2.823 & $0.817-9.759$ & 0.1010 \\
\hline Peripheral venous catheter & 6 & $(20.7)$ & 5 & $(18.5)$ & 1.0000 & 0.871 & $0.232-3.272$ & 0.8385 \\
\hline Thoracentesis & 1 & $(3.45)$ & 5 & $(18.5)$ & 0.0959 & 6.363 & $0.692-58.486$ & 0.1021 \\
\hline Paracentesis & 5 & $(17.2)$ & 2 & $(7.4)$ & 0.4239 & 0.384 & $0.068-2.172$ & 0.2790 \\
\hline Port A & 2 & $(6.9)$ & 4 & $(14.8)$ & 0.4141 & 2.348 & $0.393-14.006$ & 0.3490 \\
\hline Prior operations within 30 days & 12 & $(41.4)$ & 10 & $(37.0)$ & 0.7895 & 0.833 & $0.284-2.442$ & 0.7396 \\
\hline \multicolumn{9}{|l|}{ Pitt bacteremia score } \\
\hline Critical (Pitt score $\geq 4$ ) & 12 & $(41.4)$ & 25 & $(92.6)$ & 0.0001 & 17.71 & $3.51-89.37$ & 0.0005 \\
\hline \multicolumn{9}{|l|}{ APACHE score } \\
\hline$\geq 17$ & 15 & $(51.7)$ & 22 & $(81.5)$ & 0.0252 & 4.11 & $1.22-13.83$ & 0.0226 \\
\hline \multicolumn{9}{|l|}{ Severity by SIRS criteria } \\
\hline With septic shock & 5 & $(17.2)$ & 17 & $(63.0)$ & 8.359E04 & 8.160 & $2.361-28.207$ & 0.0009 \\
\hline Without septic shock & 24 & $(82.8)$ & 10 & $(37.0)$ & & & & \\
\hline \multicolumn{9}{|l|}{ Antibiotics treatment } \\
\hline \multicolumn{9}{|l|}{ Initial treatment alone } \\
\hline Ampicillin/sulbactam & 7 & $(24.1)$ & 4 & $(14.8)$ & 0.5064 & 0.55 & $0.14-2.13$ & 0.3842 \\
\hline Carbapenems + ampicillin/sulbactam ( \pm aminoglycosides $)$ & 4 & $(13.8)$ & 4 & $(14.8)$ & 1.0000 & 1.09 & $0.24-4.86$ & 0.9130 \\
\hline Carbapenems + aminoglycosides ( \pm ampicillin/sulbactam) & 3 & $(10.3)$ & 5 & $(18.5)$ & 0.4620 & 1.97 & $0.42-9.19$ & 0.3882 \\
\hline \multicolumn{9}{|l|}{ Definite treatment } \\
\hline Ampicillin/sulbactam & 13 & $(44.8)$ & 9 & $(33.3)$ & 0.4226 & 0.62 & $0.21-1.82$ & 0.3802 \\
\hline Carbapenems + ampicillin/sulbactam ( \pm aminoglycosides $)$ & 5 & $(17.2)$ & 4 & $(14.8)$ & 1.0000 & 0.84 & $0.20-3.50$ & 0.8050 \\
\hline Carbapenems + aminoglycosides ( \pm ampicillin/sulbactam) & 9 & $(31.0)$ & 10 & $(37.0)$ & 0.7789 & 1.31 & $0.43-3.96$ & 0.6358 \\
\hline Initial and definite treatment together & & & & & & & & \\
\hline
\end{tabular}


Table 2 (continued)

\begin{tabular}{|c|c|c|c|c|c|c|c|c|}
\hline \multirow[t]{2}{*}{ Variables } & \multicolumn{2}{|c|}{ Alive, $n=29$} & \multicolumn{2}{|c|}{ Dead, $n=27$} & \multirow[t]{2}{*}{$P^{\mathrm{a}}$} & \multicolumn{3}{|c|}{ Logistic regression } \\
\hline & No. & $(\%)$ & No. & $(\%)$ & & OR & $95 \%$ confidence interval & $P$ \\
\hline Ampicillin/sulbactam & 7 & (24.1) & 4 & $(14.8)$ & 0.5064 & 0.55 & $0.14-2.13$ & 0.3842 \\
\hline Carbapenems + ampicillin/sulbactam ( \pm aminoglycosides) & 3 & $(10.3)$ & 4 & $(14.8)$ & 0.7004 & 1.51 & $0.31-7.46$ & 0.6150 \\
\hline Carbapenems + aminoglycosides (ampicillin/sulbactam) & 2 & $(6.9)$ & 5 & $(18.5)$ & 0.2442 & 3.07 & $0.54-17.37$ & 0.2050 \\
\hline
\end{tabular}

${ }^{a}$ Chi-square test and Fisher's exact test.

No regimen has been demonstrated to be best for ERABB patients. Not surprisingly, then, the 56 patients had received various kinds of antibiotic treatments. All patients received antibiotics immediately after onset of ERABB. Carbapenems, penicillins, and aminoglycosides were the 3 classes of antibiotics used most frequently in both initial and definite treatments. However, most of the patients received treatment with a combination of antibiotics. The proportion of patients treated with a regimen of antibiotics containing ampicillin/sulbactam, or carbapenem + ampicillin/sulbactam, or carbapenem plus an aminoglycoside are shown in Fig. 1. For initial treatment, carbapenem plus an aminoglycoside and carbapenem plus ampicillin/sulbactam were the most frequently used regimens. For definite treatment, carbapenem plus an aminoglycoside was the most frequently used combination regimen. There was no significant difference in age, sex, underlying diseases, immune status, and severity of ERABB (determined by the Pitt bacteremia score and the APACHE II score) among patients receiving different treatment regimens (data not shown).

After ERABB onset, 10 patients (17.9\%) developed new respiratory failure. Nine $(16.1 \%)$ developed renal failure. Twenty-five (44.6\%) developed superimposed bacterial infections after ERABB. The respiratory tract was the most common infection focus being noted in 19 of the 25 patients with superimposed infections. All-cause mortality rates were, respectively, $23.2 \%, 30.4 \%, 37.5 \%, 48.2 \%$, and $60.7 \%$ on days $2,7,15,30$, and at discharge. The cumulative curve of mortality is shown in Fig. 2.

Comparisons for various parameters between patients who were alive and who were dead by day 30 after ERABB are listed in Table 2. For each parameter, we considered the patient to be positive for the parameter if they were positive before ERABB or if they became positive within 7 days after onset of ERABB. The parameters that differed significantly (by $\chi^{2}$ test or Fisher's exact test) between these 2 groups included the severity of patients' condition at the onset of ERABB (by APACHE II score), severity of ERABB by Pitt

Table 3

Multivariate analyses of prognostic factors for day 30 mortality in ERABB patients by multivariate logistic regression

\begin{tabular}{llll}
\hline Variables & Odds ratio & $95 \%$ confidence interval & $P$ \\
\hline $\begin{array}{l}\text { Critical (Pitt bacteremia } \\
\quad 18.53\end{array}$ & $3.10-110.72$ & 0.0014 \\
$\begin{array}{l}\text { score } \geq 4) \\
\quad \text { immunosuppression }\end{array}$ & 8.06 & $1.87-34.84$ & 0.0052 \\
\hline
\end{tabular}

bacteremia score, as well as systemic inflammatory response syndrome (SIRS) criteria, cerebrovascular accident, shock status, respiratory failure, acute renal failure, immunosuppression, usage of immunosuppressive therapy, DIC, neutropenia, hypoalbuminemia, and presence of arterial catheter during the onset of ERABB.

Significant predictors for mortality by day 30 by univariate logistic regression analysis were i) the severity of ERABB either by Pitt bacteremia score dichotomized into critical status or not, or SIRS criteria dichotomized into septic shock status or not; ii) the severity of illness at the onset of ERABB (APACHE II score, treated as the dichotomous variable); iii) cerebrovascular accident; iv) shock status; v) respiratory failure; vi) abnormal renal function; vii) acute renal failure; viii) immunosuppression; ix) usage of immunosuppressive therapy; x) DIC; xi) hypoalbuminemia; and xii) presence of arterial catheters during the onset of ERABB.

By stepwise logistic regression multivariate analysis, only severity of ERABB by Pitt bacteremia score and immunosuppression status were independent predictors for day 30 mortality of ERABB patients. Treatment regimen, no matter considering initial treatment alone, definite treatment alone, or initial and definite treatments together, was not a predictor for day 30 mortality (Table 3 ).

\section{Discussion}

Infections caused by multidrug-resistant $A$. baumannii (MDRAB) are of great concern worldwide. Many studies concerning MDRAB infections have been reported, including MDRAB bacteremia (Lai et al., 1999; Manikal et al., 2000; Smolyakov et al., 2003; Go et al., 1994). However, few of them have such extensive resistance to all antipseudomonal agents as the ones we described above, and most previous studies had a mixed study population with various kinds of multidrug resistance, and probably had both monomicrobial and polymicrobial infections. To prevent these confounding influences, we selected for our study only those patients with homogenous resistance patterns and only those with monomicrobial bacteremia. Our study results demonstrate that ERABB was associated with a high mortality rate. Severity of ERABB by Pitt bacteremia score and immunosuppression status were the only 2 independent factors that predicted day 30 mortality from ERABB. Currently available treatment regimens do not seem to have any influence on the prognosis of ERABB patients. 
The age distribution, male-to-female ratio in our patients, and distribution of underlying diseases are similar to previous studies of MDRAB bacteremia (Kuo et al., 2003; Tsai and Chen, 2003; Garcia-Garmendia et al., 2001; Cisneros and Rodriguez-Bano, 2002). In addition, all ERABB infections in the present study were nosocomially acquired, and most developed bacteremia while in ICUs, which means that these patients were critically ill while contracting ERABB. These results are also similar to prior reports (71-73.3\%) (Lai et al., 1999; Kuo et al., 2003; Cisneros et al., 1996).

In our study, most episodes of ERABB were classified as a primary bacteremia. For those cases classified as a secondary bacteremia, the respiratory tract was the most common primary focus. These findings are also similar to previous reports concerning bacteremia caused by $A$. baumannii (Lai et al., 1999; Allen et al., 2000; Cisneros et al., 1996; Seifert et al., 1995; Cisneros and RodriguezBano, 2002). The reason why patients without identifiable foci account for most of the patients with A. baumannii bacteremia is still unclear. Some authors proposed that intestinal origin leading to bacterial translocation, which is a phenomenon commonly seen in critically ill patients, might be important in the pathogenesis leading to A. baumannii bacteremia of unknown origin (Cisneros et al., 1996; Timsit et al., 1993).

In the present study, we used all-cause mortality on day 30 (after ERABB onset) as the end point for evaluating ERABB prognosis. Theoretically, mortality directly due to A. baumannii bacteremia is more representative of prognosis (Kuo et al., 2003; Cisneros et al., 1996). However, it is difficult to determine the direct cause of mortality precisely in these complicated cases, especially in the setting of retrospective analysis. In addition, all-cause day 30 mortality, not mortality at discharge, has been used in many studies investigating outcomes of bacteremia caused by various pathogens (Mylotte and Tayara, 2000; Kang et al., 2003; Kang et al., 2004), and most previous studies concerning the outcome of bacteremia caused by A. baumannii used the crude mortality rate (mortality rate evaluated at discharge) as the outcome for analysis (Kuo et al., 2003; Cisneros et al., 1996; Smolyakov et al., 2003; Blot et al., 2003). The crude mortality rate for ERABB at discharge in the present study was $60.7 \%$, which is higher than those reported in previous studies (17-46\%) (Lai et al., 1999). Disparities in microbiologic characteristics, especially in term of drug resistance and virulence, might contribute to this difference. However, the difference in crude mortality rate might also be due to the severity of underlying diseases in our patients.

Some authors reported that carbapenem plus an aminoglycoside, carbapenem plus ampicillin/sulbactam, or ampicillin/sulbactam alone seemed to be effective in the treatment of MDRAB infections, including those caused by carbapenem-resistant strains (Wang et al., 2004a, 2004b; Choi et al., 2004). Therefore, these regimens were commonly used in our patients with ERABB. However, it seems that these antibiotic regimens did not have any significant influence on the prognosis in our present retrospective study. By multivariate logistic regression analysis in determining predictors for day 30 mortality and using 3 models based on 3 different methods of grouping patients according to the treatment regimens, we identified only 2 independent predictors (severity of ERABB by Pitt bacteremia score and presence of immunosuppression). Considering initial treatment alone, or definite treatment alone, or initial and definite treatments together, whether they included ampicillin/sulbactam or not, or carbapenem plus ampicillin/sulbactam or not, or carbapenem plus aminoglycosides or not, no any one treatment regimen had statistically significant influence on day 30 mortality (Table 3). One previous study of ERABB patients, but with a smaller study population, indicated that underlying heart disease, shock, need of resuscitation, acute respiratory distress syndrome, mechanical ventilation, and multiple organ dysfunction score were significant predictors (by univariate analysis) for ERABB-associated mortality (Kuo et al., 2003). They also found that treatment regimen did not have any influence on the outcome.

We can think of 3 interpretations why treatment regimens are not an independent factor affecting prognosis of ERABB. First, the prognosis of ERABB was mainly dependent on the host condition, such as severity of disease and immune status, and thus, the antibiotic treatment did not play a role. Second, all individual antibiotics were not active against ERAB isolates. Therefore, no differences could be found even between different combinations. Third, it was possible that the day 30 mortality might not reflect the effect of treatment. However, we had evaluated the predictive variables for day 15 all-cause mortality using multivariate logistic regression model. Severity of ERABB by Pitt bacteremia score and immunosuppression status were still the only 2 independent variables predictive for day 15 mortality ( $P$ value $<0.001$ and 0.006 , respectively). To clarify these 3 possibilities, we need further studies.

The Pitt bacteremia score evaluated patients along 5 dimensions, including body temperature, blood pressure/ shock status, ventilator dependence, cardiac arrest, and consciousness at the onset of ERABB. Scoring is easy, is validated, and correlates well with clinical outcomes in studies of bloodstream infections such as Pseudomonas aeruginosa, Enterobacter, Klebsiella, pneumococcus, and others (Yu et al., 2003; Hill et al., 2001). Our study demonstrated that the Pitt bacteremia score is an independent predictive factor for day 30 mortality of patients with ERABB.

There are limitations in our study. First, the number of cases included in this study was small. This was inevitable because this kind of ERAB is a new emerging strain of MDRAB, and we want to have homogeneity of the study group for analysis. Second, this study was a retrospective study. It was therefore difficult to identify the source of ERABB in some patients, it was inevitable that there would be some incomplete data, and there were no fixed treatment 
regimens. Third, only adult patients were enrolled in this study. So the results of this study probably cannot be applied to children and adolescents with ERABB.

Because no definitely effective regimen has been found for ERABB patients and there is a high mortality rate, prevention of ERABB becomes the most important thing we can do currently. In addition to strict adherence to contact isolation measures to prevent transmission of ERAB by health care workers, previous work showed that decreased usage of broad-spectrum antibiotics, especially ceftazidime, meropenem, and imipenem, might also play a role in reducing ERAB transmission (Tsai et al., 2003).

We conclude that severity of illness at the onset of ERABB and presence of immunosuppression are the only 2 significant predictive factors for day 30 mortality. Currently available antibiotic regimens do not affect clinical outcomes of patients with ERABB.

\section{References}

Allen DM, Hartman BJ, Bennet JE, Mandell GL, Dolin R (2000) Acinetobacter Species. Mandell, Douglas, and Bennett's Principles and Practice of Infectious Disease. 2339-2344.

American College of Chest Physician/Society of Critical Care Medicine (1992) American College of Chest Physician/Society of Critical Care Medicine Consensus Conference: definitions for sepsis and organ failure and guidelines for the use of innovative therapies in sepsis. Crit Care Med 20:864-874.

Blot S, Vandewoude K, Colardyn F (2003) Nosocomial bacteremia involving Acinetobacter baumannii in critically ill patients: a matched cohort study. Intensive Care Med 29:471-475.

Caballero-Granado FJ, Becerril B, Cuberos L, Bernabeu M, Cisneros JM, Pachon J (2001) Attributable mortality rate and duration of hospital stay associated with enterococcal bacteremia. Clin Infect Dis 32:587-594.

Chang SC, Chen YC, Luh KT, Hsieh WC (1995) In vitro activities of antimicrobial agents, alone and in combination, against Acinetobacter baumannii isolated from blood. Diagn Microbiol Infect Dis 23:105-110.

Choi JY, Park YS, Cho CH, Park YS, Shin SY, Song YG, Yong D, Lee K, Kim JM (2004) Synergic in-vitro activity of imipenem and sulbactam against Acinetobacter baumannii. Clin Microbiol Infect 10:1098-1101.

Chow JW, Yu VL (1999) Combination antibiotic therapy versus monotherapy for gram-negative bacteraemia: a commentary. Int J Antimicrob Agents 11:7-12.

Cisneros JM, Reyes MJ, Pachon J, Becerril B, Caballero FJ, GarciaGarmendia JL, Ortiz C, Cobacho AR (1996) Bacteremia due to Acinetobacter baumannii: epidemiology, clinical findings, and prognostic features. Clin Infect Dis 22:1026-1032.

Cisneros JM, Rodriguez-Bano J (2002) Nosocomial bacteremia due to Acinetobacter baumannii: epidemiology, clinical features and treatment. Clin Microbiol Infect 8:687-693.

Esel D, Doganay M, Alp E, Sumerkan B (2003) Prospective evaluation of blood cultures in a Turkish university hospital: epidemiology, microbiology and patient outcome. Clin Microbiol Infect 9:1038-1044.

Galofre J, Moreno A, Mensa J, Miro JM, Gatell JM, Almela M, Claramonte X, Lozano L, Trilla A, Mallolas J, et al (1994) Analysis of factors influencing the outcome and development of septic metastasis or relapse in Salmonella bacteremia. Clin Infect Dis 18:873-878.

Garcia-Garmendia JL, Ortiz-Leyba C, Garnacho-Montero J, JimenezJimenez FJ, Perez-Paredes C, Barrero-Almodovar AE, Gili-Miner M (2001) Risk factors for Acinetobacter baumannii nosocomial bacteremia in critically ill patients: a cohort study. Clin Infect Dis 33:939-946.
Go ES, Urban C, Burns J, Kreiswirth B, Eisner W, Mariano N, MosinkaSnipas K, Rahal JJ (1994) Clinical and molecular epidemiology of acinetobacter infections sensitive only to polymyxin B and sulbactam. Lancet 344:1329-1332.

Hill PC, Birch M, Chambers S, Drinkovic D, Ellis-Pegler RB, Everts R, Murdoch D, Pottumarthy S, Roberts SA, Swager C, Taylor SL, Thomas MG, Wong CG, Morris AJ (2001) Prospective study of 424 cases of Staphylococcus aureus bacteraemia: determination of factors affecting incidence and mortality. Intern Med J 31:97-103.

Ho KK, Anderson KM, Kannel WB, Grossman W, Levy D (1993) Survival after the onset of congestive heart failure in Framingham Heart Study subjects. Circulation 88:107-115.

Hsueh PR, Chen ML, Sun CC, Chen WH, Pan HJ, Yang LS, Chang SC, Ho SW, Lee CY, Hsieh WC, Luh KT (2002) Antimicrobial drug resistance in pathogens causing nosocomial infections at a university hospital in Taiwan, 1981-1999. Emerg Infect Dis 8:63-68.

Hsueh PR, Teng LJ, Chen CY, Chen WH, Yu CJ, Ho SW, Luh KT (2002) Pandrug-resistant Acinetobacter baumannii causing nosocomial infections in a university hospital Taiwan. Emerg Infect Dis 8:827-832.

Kang CI, Kim SH, Kim HB, Park SW, Choe YJ, Oh MD, Kim EC, Choe KW (2003) Pseudomonas aeruginosa bacteremia: risk factors for mortality and influence of delayed receipt of effective antimicrobial therapy on clinical outcome. Clin Infect Dis 37:745-751.

Kang CI, Kim SH, Park WB, Lee KD, Kim HB, Oh MD, Kim EC, Choe KW (2004) Bloodstream infections caused by Enterobacter species: predictors of 30-day mortality rate and impact of broad-spectrum cephalosporin resistance on outcome. Clin Infect Dis 39:812-818.

Knaus WA, Draper EA, Wagner DP, Zimmerman JE (1985) APACHE II: a severity of disease classification system. Crit Care Med 13:818-829.

Kuo LC, Yu CJ, Lee LN, Wang JL, Wang HC, Hsueh PR, Yang PC (2003) Clinical features of pandrug-resistant Acinetobacter baumannii bacteremia at a university hospital in Taiwan. J Formos Med Assoc 102:601-606.

Lai SW, Ng KC, Yu WL, Liu CS, Lai MM, Lin CC (1999) Acinetobacter baumannii bloodstream infection: clinical features and antimicrobial susceptibilities of isolates. Kaohsiung J Med Sci 15:406-413.

Lautenbach E, Patel JB, Bilker WB, Edelstein PH, Fishman NO (2001) Extended-spectrum beta-lactamase-producing Escherichia coli and Klebsiella pneumoniae: risk factors for infection and impact of resistance on outcomes. Clin Infect Dis 32:1162-1171.

Lee CM, Lim HK, Liu CP, Tseng HK (2005) Treatment of pan_drug resistant Acinetobacter baumannii. Scand J Infect Dis 37:195-199.

Levin AS, Levy CE, Manrique AE, Medeiros EA, Costa SF (2003) Severe nosocomial infections with imipenem-resistant Acinetobacter baumannii treated with ampicillin/sulbactam. Int J Antimicrob Agents 21:58-62.

Linden PK, Pasculle AW, McDevitt D, Kramer DJ (1997) Effect of quinupristin/dalfopristin on the outcome of vancomycin-resistant Enterococcus faecium bacteraemia: comparison with a control cohort. $J$ Antimicrob Chemother 39(Suppl):145-151.

Manikal VM, Landman D, Saurina G, Oydna E, Lal H, Quale J (2000) Endemic carbapenem-resistant Acinetobacter species in Brooklyn, New York: citywide prevalence, interinstitutional spread, and relation to antibiotic usage. Clin Infect Dis 31:101-106.

Mylotte JM, Tayara A (2000) Staphylococcus aureus bacteremia: predictors of 30-day mortality in a large cohort. Clin Infect Dis 31:1170-1174.

National Committee for Clinical Laboratory Standards (2000) Methods for dilution antimicrobial susceptibility tests for bacteria that grow aerobically; approved standards-fifth edition. M7-A4. Wayne, PA: The Committee.

Seifert H, Strate A, Pulverer G (1995) Nosocomial bacteremia due to Acinetobacter baumannii. Clinical features, epidemiology, and predictors of mortality. Medicine (Baltimore) 74:340-349.

Smolyakov R, Borer A, Riesenberg K, Schlaeffer F, Alkan M, Porath A, Rimar D, Almog Y, Gilad J (2003) Nosocomial multi_drug resistant Acinetobacter baumannii bloodstream infection: risk factors and outcome with ampicillin-sulbactam treatment. $J$ Hosp Infect 54:32-38. 
Tilley PA, Roberts FJ (1994) Bacteremia with Acinetobacter species: risk factors and prognosis in different clinical settings. Clin Infect Dis 18:896-900.

Timsit JF, Garrait V, Misset B, Goldstein FW, Renaud B, Carlet J (1993) The digestive tract is a major site for Acinetobacter baumannii colonization in intensive care unit patients. J Infect Dis 168:1336-1337.

Tsai H-T, Chen C-J, Chang S-C (2003) A Case-Control Study on Association between Antimicrobial Usage and Nosocomial Infection of Pandrug- Resistant Acinetobacter baumannii in Intensive Care Units. Taipei: Graduate Institute of Epidemiology, College of Public Health, National Taiwan University.

Wang FD, Lin ML, Lee WS, Liu CY (2004) In vitro activities of beta-lactam antibiotics alone and in combination with sulbactam against Gramnegative bacteria. Int J Antimicrob Agents 23:590-595.
Wang JT, Sheng WH, Chen MY, Fang CT, Hsieh SM, Hsueh PR, Hung CC, Chang SC (2004) Nosocomial bloodstream infection in human immunodeficiency virus-infected patients in Taiwan: descriptive epidemiology and risk factors for mortality. J Formos Med Assoc 103:743-748.

Wang SH, Sheng WH, Chang YY, Wang LH, Lin HC, Chen ML, Pan HJ, Ko WJ, Chang SC, Lin FY (2003) Healthcare-associated outbreak due to pan-drug resistant Acinetobacter baumannii in a surgical intensive care unit. $J$ Hosp Infect 53:97-102.

Yu VL, Chiou CC, Feldman C, Ortqvist A, Rello J, Morris AJ, Baddour LM, Luna CM, Snydman DR, Ip M, Ko WC, Chedid MB, Andremont A, Klugman KP (2003) An international prospective study of pneumococcal bacteremia: correlation with in vitro resistance, antibiotics administered, and clinical outcome. Clin Infect Dis 37:230-237. 